\title{
Wet-Chemical Synthesis of Chiral Colloids
}

Samia Ouhajji, ${ }^{*}{ }^{\dagger}$ B⿺ Bas G. P. van Ravensteijn, ${ }^{\dagger}{ }^{\circledR}$ Carla Fernández-Rico, ${ }^{\dagger}$ Kanvaly S. Lacina, ${ }^{\dagger}$ Albert P. Philipse, ${ }^{\dagger}$ and Andrei V. Petukhov* ${ }^{* \dagger, \ddagger(0)}$

†Van 't Hoff Laboratory for Physical and Colloid Chemistry, Debye Institute for Nanomaterials Science, Utrecht University, Padualaan 8, $3584 \mathrm{CH}$ Utrecht, The Netherlands

${ }^{\ddagger}$ Laboratory of Physical Chemistry, Eindhoven University of Technology, 5600 MB Eindhoven, The Netherlands

\section{Supporting Information}

ABSTRACT: We disclose a method for the synthesis of chiral colloids from spontaneously formed hollow sugarsurfactant microtubes with internally confined mobile colloidal spheres. Key feature of our approach is the grafting of colloid surfaces with photoresponsive coumarin moieties, which allow for UV-induced, covalent clicking of colloids into permanent chains, with morphologies set by the colloid-to-tube diameter ratio. Subsequent dissolution of tube confinement yields aqueous suspensions that comprise bulk quantities of a variety of linear chains, including single helical chains of polystyrene colloids. These colloidal
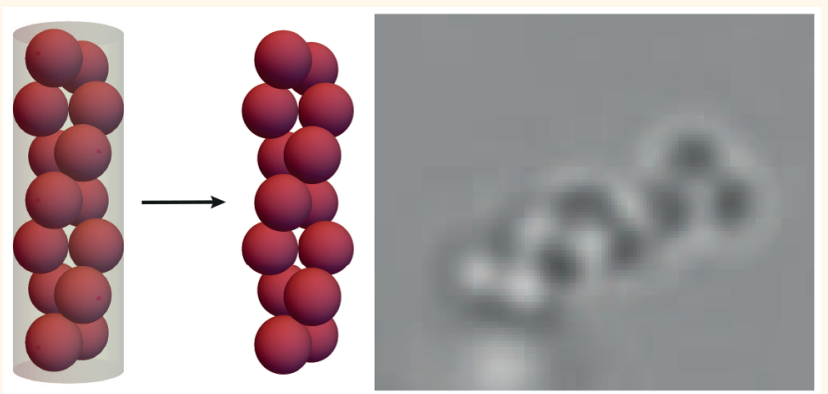
equivalents of chiral (DNA) molecules are intended for microscopic study of chiral dynamics on a single-particle level. KEYWORDS: colloidal synthesis, chirality, helices, UV irradiation, confined assembly

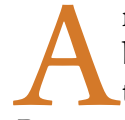
mong the captivating consequences of symmetrybreaking chirality is the coupling of rotational and translational degrees of freedom manifested in the Baranova and Zel'dovich propeller effect. ${ }^{1-4}$ Since it is not viable to visualize this intriguing coupling, and other phenomena such as chiral liquid crystal formation, on a single-particle level for chiral molecules, we set out to synthesize chiral colloids. Colloids are similar to molecules in that they are sufficiently small to exhibit Brownian motion in low-Reynolds number regime, but unlike their molecular counterparts they are in situ observable by optical microscopy. 5,6

A drawback of reported synthesis methods of chiral colloids is the need for complex, anisotropic building blocks with directional interactions. For example, colloidal helical structures that mimic the DNA helix have been assembled from anisotropic magnetic colloids ${ }^{7}$ and amphiphilic Janus spheres. $^{8}$ Furthermore, template-based (e.g., DNA) and lithographic techniques are often employed. ${ }^{2,9-11}$ These methods have several downsides such as a low yield, ${ }^{2,9}$ fragile helical products prone to dissociation, ${ }^{8}$ clusters subsisting only in an external magnetic field, ${ }^{7}$ or the need for expensive chemicals and multiple purification steps. ${ }^{10}$

Inspired by a computational study of Pickett et al., ${ }^{12}$ predicting that spheres can assemble into helical chains upon cylindrical confinement, spherical particles have been used as building blocks for complex structures. ${ }^{13,14}$ Yin and Xia, ${ }^{15}$ for example, experimentally explored the preparation of helical chains from simple colloidal spheres confined in V-grooves by capillary forces. However, the obtained structures are immobile and produced in low yield with only a limited number of particles available through slow and batch processes.

Here, we report a synthetic strategy for chiral colloids that is not limited to certain materials or particle properties and that does not require DNA, anisotropic particle shapes or directional interactions. Robust colloids have been prepared with a yield not attainable with conventional (lithography) techniques. Our method exploits the coassembly of microtubes and colloidal particles, developed in our group, to synthesize helical, zigzag and zipper colloidal chains in bulk. ${ }^{16-18}$ To this end, we designed photoresponsive colloids that form covalent bonds upon irradiation with UV-light inside the cylindrically confining environment created by the microtubes. Upon removal of the template, a variety in assemblies is obtained in aqueous suspension. The resulting colloidal chains depend sensitively on the colloid-to-tube diameter ratio.

\section{RESULTS}

Coassembly of Colloids and Microtubes. Cyclodextrins (CDs) are donutlike sugar molecules with a hydrophilic exterior and a hydrophobic interior. ${ }^{19}$ In water, the cavities of CDs can minimize undesirable interactions by incorporating the hydrophobic tails of surfactants. Mixtures of $\beta$-cyclodextrin $(\beta$-CD) and sodium dodecyl sulfate (SDS) thus form hostguest inclusion complexes in a 2:1 molar ratio at temperatures

Received: July 4, 2018

Accepted: November 14, 2018

Published: November 14, 2018 


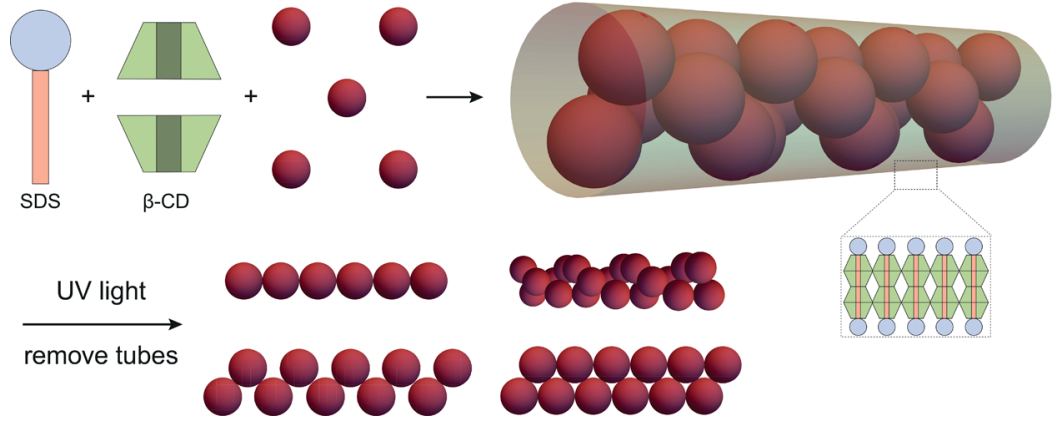

Figure 1. Schematic representation of the assembly of spheres into chains. Photoresponsive colloidal spheres are confined in (thermoreversible) microtubes that are composed of bilayers of SDS and $\beta$-CD after a heating/cooling cycle. Due to geometric restrictions, the colloids assemble in a variety of structures, including helical sphere chains, for a colloid-to-tube diameter ratio of about 0.5 . Upon irradiation with UV-light, the colloids are covalently bound together inside the microtubes. By diluting the system with water, the microtubes dissolve and stable colloidal structures, including chiral chains, in bulk are obtained.
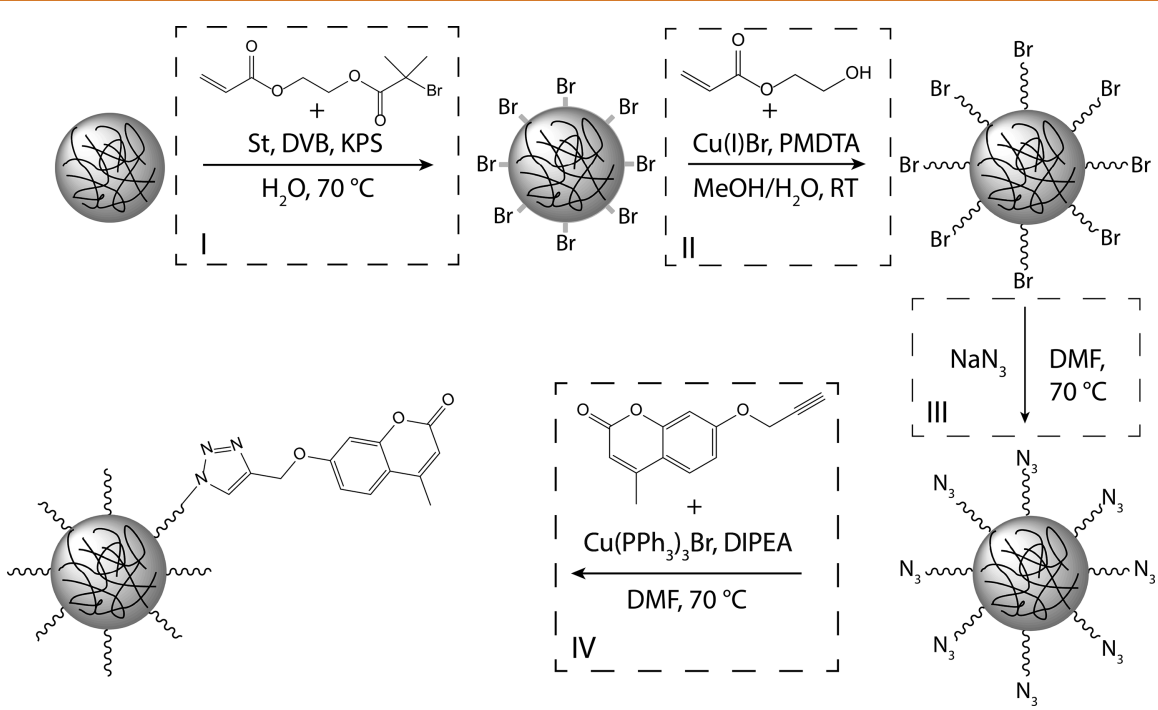

Figure 2. Schematic overview of the synthesis route toward photoresponsive colloidal particles. (I) Seeded emulsion polymerization of crosslinked polystyrene particles in the presence of 2-(2-bromoisobutyryloxy)ethyl acrylate (BIEA), styrene (St), and divinylbenzene (DVB). (II) Polymeric 2-hydroxyethyl acrylate (HEA) hairs are grafted from the surface of brominated colloidal initiators using SI-ATRP. (III) Halogen chain ends of $\mathbf{p}$ (HEA) are substituted by azides via a nucleophilic substitution reaction. (IV) Alkyne-coumarin is coupled to the azide chain ends via the Huisgen click reaction.

above $40{ }^{\circ} \mathrm{C}$ in aqueous media. Upon cooling to room temperature these SDS@2 $\beta$-CD complexes self-assemble into multiple equally spaced curved bilayers forming a set of

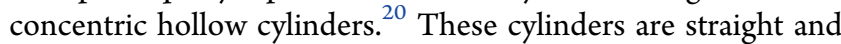
rigid tubes that can be several tens of micrometers long with a pore diameter of $0.9 \mu \mathrm{m} \pm 10 \%$. On addition of colloidal spheres, the tubular structures act as a cylindrically confining environment and ordered, chainlike colloid-in-tube assemblies are obtained. As the microtubes form a space-filling structure, all colloidal particles with a size smaller than the microtube diameter appear confined in the tubes. Depending on the size ratio of the colloid-to-tube diameters (see Figure S1 in the Supporting Information), various structures, including helical sphere chains, are formed; three distinct size ratios were chosen for further investigation.

Photoresponsive Particles. Upon removal of the confinement, the formed colloid-in-tube assemblies dissociate. To secure the morphology of colloid structures prior to dissociation, we designed photoresponsive polystyrene spheres for UV-induced cross-linking; see Figure 1. The desired sterically stabilized photoactive colloids in water were obtained by utilizing surface-initiated atom transfer radical polymerization (SI-ATRP) to graft well-defined hydrophilic polymeric hairs from the surface of polystyrene spheres. ${ }^{21,22}$ The ATRP reaction was quenched to obtain polymer brushes of desired length $(\approx 30 \mathrm{~nm})$, end-functionalized with bromine atoms. These halogen atoms could then be substituted with azides to which alkyne-coumarin ${ }^{23-26}$ molecules could be attached via the efficient and robust click chemistry pathway ${ }^{27}$ (see Figure 2).

Having successfully synthesized photo-cross-linkable colloids, as characterized with infrared spectroscopy (see Figure S2), dynamic light scattering, and microscopy techniques, the behavior in bulk was investigated. Covalent bonds between neighboring colloids were evidently formed, as the photoresponsive colloids clearly showed the expected photoinduced aggregation in bulk (see Supporting Information). The assembly into chiral structures was subsequently studied.

Assembly into Sphere Chains. Polystyrene spheres with surface-immobilized coumarin derivatives were assembled inside the microtubes with a particle loading of $10 \mathrm{wt} \%$. Upon irradiation of the photoresponsive colloids inside the 
microtubes with UV-light with a wavelength of $365 \mathrm{~nm}$, coumarin molecules on adjacent particles photo-dimerized and the colloid-in-tube assemblies were affixed. The cylindrical confinement was subsequently removed by dilution of the system with water, causing the microtubes to disassemble (see Figure 3). The obtained structures exhibit Brownian motion
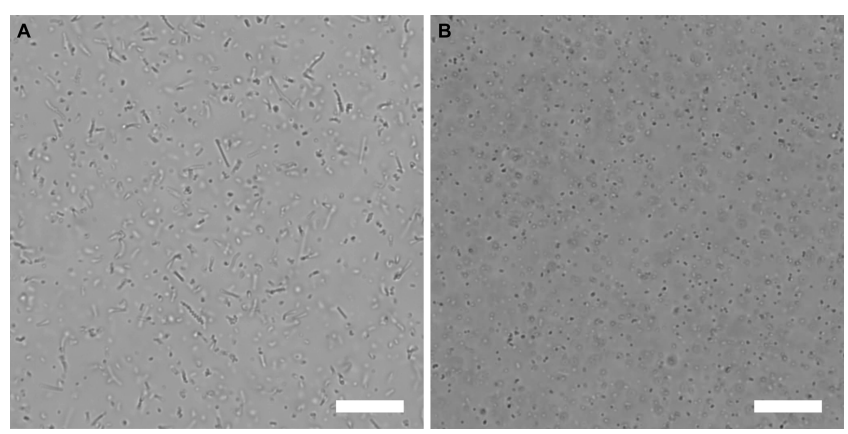

Figure 3. Optical microscopy images of colloidal dispersions upon UV irradiation and dissolution of microtubes. (a) Large number of colloidal chains in aqueous suspension. Field of view is $123 \mu \mathrm{m} \times$ $123 \mu \mathrm{m}$. (b) Control sample that was enclosed in aluminum foil to prevent the penetration of UV-light; no assemblies are formed. Scale bars are $10 \mu \mathrm{m}$.

(see Supporting Videos) and are mechanically robust as the assemblies can withstand hours of exposure to ultrasonication without disintegrating. Per synthesis cycle about $10^{9}$ chains are obtained (see the Supporting Information for calculation of overall yield).

Analysis of Formed Structures. The size ratio of the colloid-to-tube diameters ultimately determines the structures that can be formed. To investigate the effect of the size ratio on the assemblies, we employed colloids of different sizes. Figure 4 shows the distribution of structures that are obtained as a function of the colloid-to-tube diameter size ratio. As the particle diameter approaches that of the microtubes (colloidto-tube diameter ratio of about one), a clear preference for assembly in linear, rod-like chains is observed. Particles that are much smaller than the microtube diameter, do not feel the

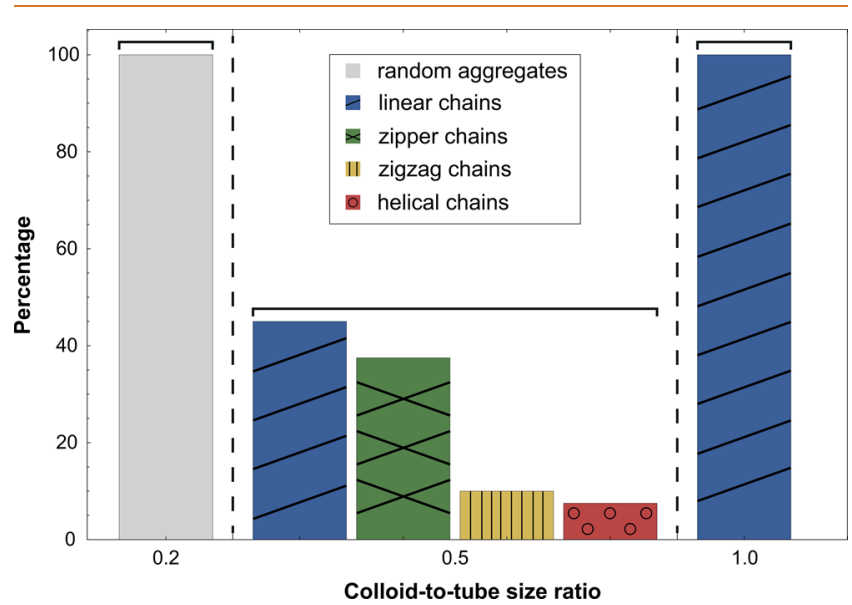

Figure 4. Probability to find a cluster of a certain shape. Distribution of linear, zigzag, zipper, and helical chains as a function of the colloid-to-tube diameter size ratio. The percentages were determined by counting at least 100 assemblies per size ratio as found in representative microscopy images. confinement and are thus randomly distributed throughout the cylindrical tubes forming small and random aggregates. Conversely, particles that are much larger than one micron cannot be incorporated inside the tubes. The structures in bulk resulting from particles that have a colloid-to-tube diameter ratio of about 0.5 vary from linear chains, zigzag and zipper chains to helical assemblies; see Figure 5, Figure S7, and
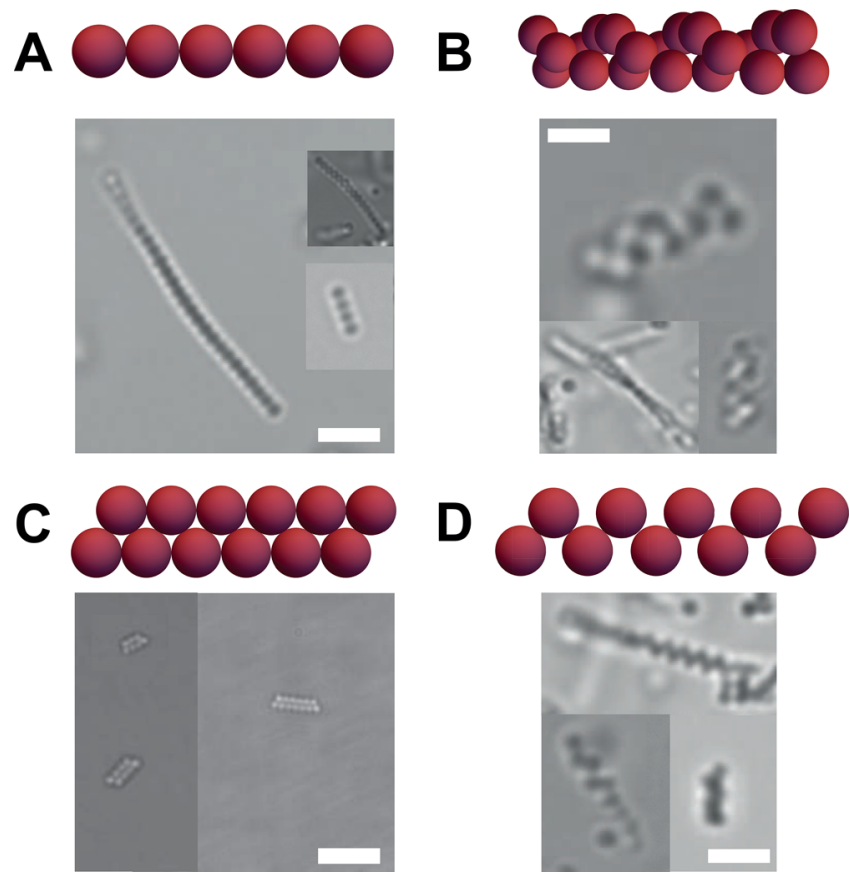

Figure 5. Optical microscopy images. (a) Linear sphere chain, (b) helical chain, (c) zipper chain, and (d) zigzag sphere chain. Insets show models of the obtained structures. Scale bars are $10 \mu \mathrm{m}$. See also Supporting Videos.

Supporting Videos. Typically, a racemic mixture is formed and the chain lengths vary from 2 (dimers) to 30 spheres per chain, with an average chain length of 10 spheres. The average length of the chains can be varied by changing the weight percentage of particles inside the microtubes. An increase in the particle concentration leads to an increase in the number of spheres per chain, but the length is ultimately limited by the extent of the tubes, typically in the range of tens of microns.

\section{DISCUSSION}

By utilizing microtubes and photoresponsive polystyrene spheres, freely dispersed complex structures in bulk are obtained. As microtubes tend to be sensitive to changes in concentration, $\mathrm{pH}$, and ionic strength, among others, we developed photoresponsive colloids since light is an external and nondisruptive trigger toward the confinement. The advantage of employing coumarin as the photoresponsive glue is that no photoinitiator was required. However, coumarin is hydrophobic and grafting densities had to be kept to a minimum to safeguard colloidal stability and to prevent intraparticle coumarin cross-linking. On the other hand, the surface number density of coumarin molecules had to be sufficiently high to induce colloid attachment.

A wide range of structures is produced for a colloid-to-tube diameter ratio of about 0.5 . According to theory, the yield of each species and the pitch of the chiral chains could be 
controlled by fine-tuning the ratio of the colloid-to-tube diameters. ${ }^{12}$ As a consequence of the inherent polydispersity in particle size and microtube diameter, however, it is difficult to fall within these narrow ranges experimentally. Therefore, postsynthesis separation techniques were explored. Established chiral separation methods for molecules, such as chromatography and capillary electrophoresis, are not suitable for colloidal particles as they utilize chirality specific chemical interactions. ${ }^{28}$ We employed density gradient centrifugation (DGC) to successfully separate small clusters and single particles from the desired chains. Unfortunately, DGC cannot distinguish between a linear or helical chain composed of 10 spheres. Our chiral colloids might be isolated in a helical flow flied; Aristov et al. demonstrated the separation of left- and right-handed micron-sized chiral particles in a helical fluid flow inside a microfluidic device. ${ }^{29}$

Since the colloidal assemblies show variations in size, geometry, and type of enantiomers, they are ideal for singleparticle studies of the dynamics and Brownian motion of colloids with chiral and other geometries, which is currently ongoing work. Furthermore, preliminary results have shown that the synthesis procedure as outlined in this Article is not limited by the chemical nature of the colloids. Photoresponsive silica spheres have been prepared in a similar fashion yielding colloidal silica assemblies. By using metallic functionalization, chiral plasmonic properties can be achieved for the fabrication of optically active media. ${ }^{30}$ In addition, our wet synthesis method can easily be performed on larger scales further increasing the yield. Finally, the chiral structure of the microtubes, as a result of the constituent chiral cyclodextrin molecules, could be exploited to synthesize homochiral chains. However, the chiral coupling is currently too weak to influence the confined colloids. If the chirality on the nanoscale could be enhanced, this might lead to homochiral chains but this is a topic for future research.

\section{CONCLUSIONS}

In conclusion, we have developed a synthesis method to produce robust colloidal assemblies, including chiral colloids, in high yield. Employing the coassembly of colloidal particles and microtubes, colloidal helices can be assembled from spherical building blocks. Our synthesis route yields photocross-linkable colloidal polystyrene spheres that are sterically stabilized with polymer hairs and end-functionalized with coumarin molecules. By exposure to UV-light, the coumarin molecules act as a chemical glue holding the spheres in place even after dissolution of confining tubes. Eventually, a library of ordered structures, such as linear chiral assemblies, zigzag, and zipper configurations of isotropic colloidal spheres, has become available in bulk. These colloidal analogues of chiral molecules are a promising model system to study the role of chirality in the self-assembly and the diffusion of biological macromolecules, such as the DNA helix. Further insight into the functionality of chirality on the molecular scale could aid in the design of chiral materials.

\section{METHODS}

Materials. Styrene (St, 99\%), divinylbenzene (DVB, 55\% mixture of isomers, tech. grade), 2-bromoisobutyryl bromide (BiBB, 98\%), 2hydroxyethyl acrylate (HEA, 96\%, contains 200-650 ppm monomethyl ether hydroquinone as inhibitor), sodium sulfate $\left(\mathrm{Na}_{2} \mathrm{SO}_{4}\right.$, ACS reagent, $\geq 99 \%$, anhydrous), copper bromide $(\mathrm{Cu}(\mathrm{I}) \mathrm{Br}, 98 \%$, stored under inert atmosphere), $N, N, N^{\prime}, N^{\prime}, N^{\prime \prime}$-pentamethyldiethyle- netriamine (PMDTA, 99\%), dimethylformamide (DMF, $\geq 99 \%$ ), bromotris(triphenylphosphine) copper(I) $\left(\mathrm{Cu}\left(\mathrm{PPh}_{3}\right)_{3} \mathrm{Br}, 98 \%\right), \mathrm{N}, \mathrm{N}$ diisopropyl-ethylamine (DIPEA, $\geq 98 \%$ ), propargyl bromide (Prp-Br, $80 \mathrm{wt} \%$ solution in toluene), sodium dodecyl sulfate (SDS, >97\%), and $\beta$-cyclodextrin $(\beta$-CD, $\geq 97 \%)$ were obtained from Sigma-Aldrich. Potassium persulfate (KPS, $>99 \%$ for analysis), sodium bisulfite ( $\mathrm{NaHSO}_{3}$, ACS reagent), pyridine (>99\%), and 7-hydroxy-4methylcoumarin (97\%) were purchased from Acros Organics. Methanol ( $\mathrm{MeOH}$, exceeds ACS specifications) was obtained from J.T. Baker, and dichloromethane (DCM, peptide synthesis) was obtained from Biosolve. Sodium azide $\left(\mathrm{NaN}_{3}, 99 \%\right)$ was obtained from VWR. All chemicals were used as received except for $\beta$-CD which was dried under a heat lamp prior to use to remove excess water. The water used for all syntheses was purified using a Milli-Q water purification system.

Synthesis of 2-(2-Bromoisobutyryloxy)ethyl Acrylate (BIEA). The synthesis of BIEA was adapted from ref 31 . A solution of HEA $(40 \mathrm{~mL}, 348 \mathrm{mmol})$ and pyridine $(31 \mathrm{~mL}, 383 \mathrm{mmol})$ in DCM $(250$ $\mathrm{mL}$ ) was stirred and cooled in an ice bath. Under nitrogen atmosphere, a solution of $\mathrm{BiBB}(36.45 \mathrm{~mL}, 348 \mathrm{mmol})$ in DCM $(50 \mathrm{~mL})$ was added dropwise over the course of $1 \mathrm{~h}$. During the addition, a white precipitate was formed ( $\mathrm{HBr}$-pyridine). After complete addition of the $\mathrm{BiBB}$ solution, the reaction mixture was stirred an additional $3 \mathrm{~h}$ at room temperature. The precipitate was filtered off, and DCM was evaporated under reduced pressure. A yellow oil and additional precipitate was formed. The additional precipitate was filtered off and washed with DCM. The DCM from washing and the yellow oil were combined and washed with water (three times, $50 \mathrm{~mL}$ per washing step). The oil phase was dried over $\mathrm{Na}_{2} \mathrm{SO}_{4}$, and finally the DCM was evaporated under reduced pressure. The resulting yellow oil was distilled to complete the purification procedure yielding a colorless oil. ${ }^{1} \mathrm{H}$ NMR $\left(400 \mathrm{MHz}, \mathrm{CDCl}_{3}, \delta\right)$ : $6.43(\mathrm{~d}, 1 \mathrm{H}), 6.14(\mathrm{dd}, 1 \mathrm{H}), 5.85(\mathrm{~d}, 1 \mathrm{H}), 4.4(\mathrm{~s}, 4 \mathrm{H}), 1.9(\mathrm{~s}, 6 \mathrm{H})$.

Synthesis of Brominated Colloidal Initiators (CPs-Br). Crosslinked polystyrene particles (CPs) were synthesized using a standard emulsion polymerization method described in literature. ${ }^{32,33}$ A 250 $\mathrm{mL}$ round-bottom flask equipped with magnetic stir bar was placed in an oil bath at $80{ }^{\circ} \mathrm{C}$. Water $(100 \mathrm{~mL})$ was charged into the reactor and allowed to reach the bath temperature. St $(11.6 \mathrm{~mL}, 100 \mathrm{mmol})$, DVB $(0.35 \mathrm{~g}, 2.4 \mathrm{mmol})$, and SDS $(62.5 \mathrm{mg}, 0.2 \mathrm{mmol})$ dissolved in water $(25 \mathrm{~mL})$ were added. The complete mixture was allowed to heat up to the temperature of the bath. Finally, the addition of KPS $(0.39 \mathrm{~g}, 1.4 \mathrm{mmol}$ dissolved in $18.8 \mathrm{~mL}$ water) initiated the polymerization. The reaction was allowed to continue for $24 \mathrm{~h}$ at 80 ${ }^{\circ} \mathrm{C}$. The resulting latex had a solid content of $6.7 \%$ (measured gravimetrically). The obtained particles had a diameter of $386 \mathrm{~nm}$ with a polydispersity of $6.7 \%$ as determined with transmission electron microscopy (TEM). Dynamic light scattering (DLS) measurements revealed an apparent hydrodynamic diameter of 406 $\mathrm{nm}$ with a corresponding polydispersity index (PDI) of 0.036 .

The synthesized particles were used as seeds in the second step, in which BIEA was polymerized onto the surface of the prepared CPs particles. $^{22}$ To this end, crude seed dispersion $(\mathrm{CPs}, 12.5 \mathrm{~mL})$ and water $(12.5 \mathrm{~mL})$ were introduced into a $50 \mathrm{~mL}$ round-bottom flask equipped with a magnetic stir bar. The dispersion was degassed with nitrogen for $30 \mathrm{~min}$. Subsequently, a mixture consisting of St $(0.5 \mathrm{~mL}$, $4.4 \mathrm{mmol})$, DVB $(10 \mu \mathrm{L}, 0.07 \mathrm{mmol})$, and BIEA $(0.4 \mathrm{~g}, 1.5 \mathrm{mmol})$ was injected under inert atmosphere. After $5 \mathrm{~min}$ of stirring, polymerization of the newly added monomers was initiated by the addition of a degassed, aqueous KPS solution $(16 \mathrm{mg}, 0.06 \mathrm{mmol}$ in $2.5 \mathrm{~mL}$ water). The polymerization was allowed to run for $6 \mathrm{~h}$ at 70 ${ }^{\circ} \mathrm{C}$ after which the reaction was quenched by removing the flask from the oil bath. The particles were washed with water three times by means of centrifugation and redispersion cycles.

Infrared (IR) spectroscopy was used to confirm successful coupling of BIEA to the surface of the polystyrene particles (see Figure S2; $1732 \mathrm{~cm}^{-1}, \mathrm{C}=\mathrm{O}$ vibration of BIEA). DLS gave an apparent hydrodynamic diameter of $464 \mathrm{~nm}$ and a PDI of 0.095 .

Grafting of Poly(HEA) Brushes from CPs-Br Using SI-ATRP (CPs-p(HEA)-Br). Cu(I)Br (6.9 mg, $0.05 \mathrm{mmol})$ and HEA (69 $\mu \mathrm{L}$, 
$0.60 \mathrm{mmol}$ ) were mixed with $\mathrm{MeOH} / \mathrm{H}_{2} \mathrm{O}$ mixture $(7: 3, \mathrm{v} / \mathrm{v})(0.5$ $\mathrm{mL})$ in an oven-dried Schlenk flask, resulting in a light green mixture (note: the copper bromide salt does not completely dissolve in the reaction medium). The obtained mixture was degassed by evacuation and refilling with nitrogen (three cycles). Subsequently, PMDTA (29 $\mu \mathrm{L}, 0.14 \mathrm{mmol}$ ) was injected, resulting in the appearance of a blue/ green color and complete solubilization of the copper bromide salt. The degassing procedure was repeated once more to further exclude the presence of oxygen in the obtained catalyst/monomer reaction mixture.

In a separate Schlenk flask, the CPs-Br colloids dispersed in a 7:3 (v/v) $\mathrm{MeOH} / \mathrm{H}_{2} \mathrm{O}$ mixture $(0.5 \mathrm{~mL}, 2$ wt $\%$ ) were degassed by evacuation and refilling with nitrogen (three cycles). After degassing, the dispersion was injected into the monomer/catalyst mixture under inert atmosphere. The resulting reaction mixture had a white/green appearance. The ATRP reaction was allowed to run for $40 \mathrm{~min}$ at room temperature, after which the reaction was terminated by exposure to air, yielding an intense blue color. The particles were washed three times with the $\mathrm{MeOH} / \mathrm{H}_{2} \mathrm{O}$ mixture, 10 times with a 50 $\mathrm{mM}$ aqueous $\mathrm{NaHSO}_{3}$ solution and finally three times with water. The $\mathrm{NaHSO}_{3}$ solution was used to facilitate the removal of the copper catalyst. After the complete washing procedure, a stable colloidal dispersion with a solid content of $1 \%$ was obtained.

The presence of $\mathrm{p}$ (HEA) was probed using IR spectroscopy (see Figure $\mathrm{S} 2 ; 1732 \mathrm{~cm}^{-1}, \mathrm{C}=\mathrm{O}$ vibration of pending ester functionalities of grafted polymers). Furthermore, DLS showed a clear increase in apparent hydrodynamic diameter $(529 \mathrm{~nm})$, providing additional evidence for successful polymer grafting.

Nucleophilic Substitution of Halogen Chain Ends of Grafted p(HEA) Hairs with Sodium Azide (CPs-p(HEA)- $\mathrm{N}_{3}$ ). $\mathrm{NaN}_{3}$ (4.5 mg, $0.07 \mathrm{mmol}$ ) was dissolved in DMF (0.5 mL). To this solution, a dispersion containing the $\mathrm{p}(\mathrm{HEA})$ grafted colloids in DMF was added $(0.5 \mathrm{~mL}$, solid content $=1 \%)$. The obtained reaction mixture was allowed to stir for $24 \mathrm{~h}$ at $70{ }^{\circ} \mathrm{C}$. After this period, the particles were washed with DMF (three times) and water (three times) to remove excess $\mathrm{NaN}_{3}$. IR spectroscopy was used to verify successful formation of azido-end-functionalized polymers (see Figures S2; $2096 \mathrm{~cm}^{-1}$ ).

Synthesis of 4-Methyl-7-(prop-2-yn-1-yloxy)-coumarin (Alkyne-Coumarin). The synthesis was adapted from ref 34. 4-Methyl7-hydroxy-coumarin $(2.5 \mathrm{~g}, 14.3 \mathrm{mmol})$ and $\mathrm{K}_{2} \mathrm{CO}_{3}(8.18 \mathrm{~g}, 59$ mmol) were transferred into a $100 \mathrm{~mL}$ Schlenk flask containing a magnetic stir bar. The flask was subsequently evacuated and refilled with nitrogen gas three times. A separate Schlenk flask containing dry DMF (stored over freshly regenerated mole sieves of $4 \AA$ ) was evacuated and refilled with nitrogen (three cycles). Subsequently, the dried DMF $(40 \mathrm{~mL})$ was transferred to the flask containing coumarin and $\mathrm{K}_{2} \mathrm{CO}_{3}$ using a tube, yielding a clear, yellow solution after stirring for $10 \mathrm{~min}$. While stirring, propargyl bromide $(2.6 \mathrm{~mL}, 23.3 \mathrm{mmol})$ was injected via a septum resulting in a slightly darker yellow reaction mixture. Stirring was continued for $72 \mathrm{~h}$ at room temperature.

The obtained product was purified by precipitation of the crude mixture into water $(100 \mathrm{~mL})$. The solid was filtered and washed with water four times. The resulting solid was dried under vacuum for $2 \mathrm{~h}$ at $40{ }^{\circ} \mathrm{C}$ and recrystallized from methanol. The product was stored in the freezer. ${ }^{1} \mathrm{H}$ NMR $\left(400 \mathrm{MHz}, \mathrm{CDCl}_{3}, \delta\right): 7.5(\mathrm{~m}, 1 \mathrm{H}), 6.9(\mathrm{~m}$, $2 \mathrm{H}), 6.2(\mathrm{~d}, 1 \mathrm{H}), 4.8(\mathrm{~d}, 2 \mathrm{H}), 2.6(\mathrm{t}, 1 \mathrm{H}), 2.4(\mathrm{~d}, 3 \mathrm{H})$. FT-IR: $\nu=$ $3300 \mathrm{~cm}^{-1}$ (diagnostic signal used to confirm successful modification of coumarin precursor). UV-vis (ethanol): $\lambda_{\max }=320 \mathrm{~nm}$.

Coupling of Alkyne-Coumarin to CPs-p(HEA)- $\mathrm{N}_{3}$ Using Click Chemistry (CPs-p(HEA)-Coumarin). $\mathrm{Cu}\left(\mathrm{PPh}_{3}\right)_{3} \mathrm{Br}(6.25 \mathrm{mg}$, 6.7 $\mu \mathrm{mol}$ ) was introduced into a $10 \mathrm{~mL}$ round-bottom flask equipped with a magnetic stir bar. To this, DIPEA $(13 \mu \mathrm{L}, 75 \mu \mathrm{mol})$ and alkyne-coumarin $(0.5 \mathrm{~mL}$ of a DMF stock solution, concentration = $4.9 \mathrm{mg} / \mathrm{mL}, 23 \mathrm{mmol} / \mathrm{mL}$ ) were added. Finally, a dispersion containing CPs-p $(\mathrm{HEA})-\mathrm{N}_{3}$ in DMF $(0.25 \mathrm{~mL}$, solid content $=1 \%)$ was injected. The reaction was allowed to run for $24 \mathrm{~h}$ under gentle stirring at $70{ }^{\circ} \mathrm{C}$. After this period, the colloids were washed with DMF. IR spectroscopy was used to probe the effectiveness of the coumarin coupling to the chain-ends of the grafted polymers (see
Figure S2; disappearance of the signal at $2096 \mathrm{~cm}^{-1}$, free azide vibration).

Coassembly of Colloids and Microtubes. SDS and $\beta$-CD were weighed and mixed as to obtain a molar ratio of $\beta$-CD:SDS of $2: 1$ and a total concentration of SDS and $\beta$-CD of $10 \mathrm{wt} \%$ in water (typically $1.72 \mathrm{~g}$ or $1.5 \mathrm{mmol} \beta$-CD and $0.22 \mathrm{~g}$ or $0.8 \mathrm{mmol}$ SDS in $17.5 \mathrm{~g}$ $\mathrm{H}_{2} \mathrm{O}$ ). While being magnetically stirred, the mixture was heated to 60 ${ }^{\circ} \mathrm{C}$ in an oil bath until a transparent and isotropic solution was obtained. The microtubes were formed upon cooling to room temperature; a turbid and viscous suspension is obtained. The photoresponsive colloidal particles were incorporated inside the microtubes by centrifuging aqueous colloidal dispersions and replacing the supernatant by microtube suspension. Typically, the mixture of colloids and microtubes contained $10 \mathrm{wt} \%$ colloidal particles. The mixture was heated to approximately $60{ }^{\circ} \mathrm{C}$ to melt the microtubes, sonicated and then vortexed to disperse the particles inside the microtubes. Upon cooling to room temperature, the sample was gently rotated to avoid sedimentation of the colloids.

Irradiation Experiments. A $14 \mathrm{~W}$ low-pressure mercury lamp emitting UV-light with a wavelength of $365 \mathrm{~nm}$ was used to cross-link the photoresponsive colloids. Samples consisting of colloids incorporated inside microtubes in cuvettes were placed in a water bath to prevent the temperature increasing above $40{ }^{\circ} \mathrm{C}$; the melting temperature of the microtubes. Typically, samples were irradiated for $24 \mathrm{~h}$. Control samples were enclosed in aluminum foil to prevent the penetration of UV-light.

Characterization. IR spectra were obtained using a PerkinElmer FT-IR/FIR Frontier spectrometer in attenuated total reflectance (ATR) mode. The measurements were carried out on powders (obtained by drying the corresponding particle dispersion).

Transmission electron microscopy (TEM) pictures were taken with a Philips Tecnai10 electron microscope typically operating at $100 \mathrm{kV}$. Bright field images were recorded using a SIS Megaview II CCD camera. The samples were prepared by drying a drop of diluted aqueous particle dispersion on top of polymer coated copper grids.

Scanning electron microscopy (SEM) pictures were taken with a FEI XL30 FEG scanning electron microscope operating at 5-15 kV. SEM samples were prepared by drying a drop of diluted aqueous particle dispersion on top of polymer coated copper grids and sticking these grids on a stub using a conductive carbon sticker, which was coated with a platinum layer of typically $6 \mathrm{~nm}$.

Dynamic light scattering (DLS) was performed using a Malvern Zetasizer Nano instrument using highly diluted aqueous dispersions at $25{ }^{\circ} \mathrm{C}$. The DLS measurements were taken in ten runs of $10-15$ individual measurements in backscatter mode $\left(173^{\circ}\right)$. The hydrodynamic particle dimensions are reported as number-average diameters with their corresponding polydispersity index (PDI).

${ }^{1} \mathrm{H}$ NMR spectra were recorded using a Varian MRF400 $400 \mathrm{MHz}$ NMR machine. $\mathrm{CDCl}_{3}$ was employed as solvent.

UV-vis spectra were recorded on a PerkinElmer Lambda-35 spectrophotometer from $\lambda=450 \mathrm{~nm}$ to $\lambda=250 \mathrm{~nm}$ with $1.0 \mathrm{~nm}$ intervals. Helma QS quartz cuvettes with a path length of $10 \mathrm{~mm}$ were used. Sample concentrations were chosen such that the maximum absorption remained below 2.5.

Optical microscopy images were obtained using a Nikon Eclipse Ti-E inverted microscope equipped with a Hamatsu Orca Flash 4.0 V2 digital camera. A Nikon CFI Apo TIRF objective (100X magnification, N.A. 1.49) was used. Pictures were recorded in bright field mode. For fluorescence microscopy, a Nikon Intensilight CHGFI light source was used with a TRITC filter cube.

\section{ASSOCIATED CONTENT}

\section{Supporting Information}

The Supporting Information is available free of charge on the ACS Publications website at DOI: 10.1021/acsnano.8b05065.

Broad-view real-time optical microscopy video illustrating Brownian motion of particle assemblies (AVI) 
Real-time optical microscopy video illustrating Brownian motion of a linear chain (AVI)

Real-time optical microscopy video illustrating Brownian motion of a zigzag chain (AVI)

Real-time optical microscopy video illustrating Brownian motion of a zipper chain (AVI)

Real-time optical microscopy video illustrating Brownian motion of a helical chain (AVI)

Colloids at microtubes, IR spectra, behavior of the photoresponsive colloids in bulk, photochemistry of alkyne-coumarin, calculations of the estimated yield, morphology of the photoresponsive colloids, fluorescence microscopy images, SEM images (PDF)

\section{AUTHOR INFORMATION}

\section{Corresponding Authors}

*E-mail: S.Ouhajji@uu.nl.

*E-mail: A.V.Petukhov@uu.nl.

\section{ORCID}

Samia Ouhajji: 0000-0003-0142-1467

Bas G. P. van Ravensteijn: 0000-0001-9024-3927

Andrei V. Petukhov: 0000-0001-9840-6014

\section{Notes}

The authors declare no competing financial interest.

\section{ACKNOWLEDGMENTS}

The authors acknowledge The Netherlands Organisation for Scientific Research (NWO) for funding (Grant Number 712.014.002). S.O. thanks R.N. Georgiev for the drawing of a confined double helical sphere chain and J. Landman for the drawing of SDS and $\beta$-CD.

\section{REFERENCES}

(1) Baranova, N. B.; Zel'dovich, B. Y. Separation of Mirror Isomeric Molecules by Radio-Frequency Electric Field of Rotating Polarization. Chem. Phys. Lett. 1978, 57, 435-437.

(2) Schamel, D.; Pfeifer, M.; Gibbs, J. G.; Miksch, B.; Mark, A. G.; Fischer, P. Chiral Colloidal Molecules and Observation of the Propeller Effect. J. Am. Chem. Soc. 2013, 135, 12353-12359.

(3) Kraft, D. J.; Wittkowski, R.; ten Hagen, B.; Edmond, K. V.; Pine, D. J.; Löwen, H. Brownian Motion and the Hydrodynamic Friction Tensor for Colloidal Particles of Complex Shape. Phys. Rev. E 2013, 88, No. 050301

(4) Han, Y.; Alsayed, A. M.; Nobili, M.; Zhang, J.; Lubensky, T. C.; Yodh, A. G. Brownian Motion of an Ellipsoid. Science 2006, 314, 626-630.

(5) Manoharan, V. Colloidal Matter: Packing, Geometry, and Entropy. Science 2015, 349, 1253751.

(6) Poon, W. Colloids as Big Atoms. Science 2004, 304, 830-831.

(7) Zerrouki, D.; Baudry, J.; Pine, D. J.; Chaikin, P.; Bibette, J. Chiral Colloidal Clusters. Nature 2008, 455, 380-382.

(8) Chen, Q.; Whitmer, J. K.; Jiang, S.; Bae, S. C.; Luijten, E.; Granick, S. Supracolloidal Reaction Kinetics of Janus Spheres. Science 2011, 331, 199-202.

(9) Feng, W.; Kim, J. Y.; Wang, X.; Calcaterra, H. A.; Qu, Z.; Meshi, L.; Kotov, N. A. Assembly of Mesoscale Helices with Near-Unity Enantiomeric Excess and Light-Matter Interactions for Chiral Semiconductors. Sci. Adv. 2017, 3, e1601159.

(10) Siavashpouri, M.; Wachauf, C. H.; Zakhary, M. J.; Praetorius, F.; Dietz, H.; Dogic, Z. Molecular Engineering of Chiral Colloidal Liquid Crystals using DNA Origami. Nat. Mater. 2017, 16, 849-856.

(11) Yeom, J.; Santos, U. S.; Chekini, M.; Cha, M.; de Moura, A. F.; Kotov, N. A. Chiro-magnetic Nanoparticles and Gels. Science 2018, 359, 309-314.
(12) Pickett, G. T.; Gross, M.; Okuyama, H. Spontaneous Chirality in Simple Systems. Phys. Rev. Lett. 2000, 85, 3652-3655.

(13) Fu, L.; Bian, C.; Shields, C. W., IV; Cruz, D. F.; López, G. P.; Charbonneau, P. Assembly of Hard Spheres in a Cylinder: A Computational and Experimental Study. Soft Matter 2017, 13, 32963306.

(14) Li, F.; Badel, X.; Linnros, J.; Wiley, J. B. Fabrication of Colloidal Crystals with Tubular-Like Packings. J. Am. Chem. Soc. 2005, 127, 3268-3269.

(15) Yin, Y.; Xia, Y. Self-Assembly of Spherical Colloids into Helical Chains with Well- Controlled Handedness. J. Am. Chem. Soc. 2003, 125, 2048-2049.

(16) Jiang, L.; de Folter, J. W. J.; Huang, J.; Philipse, A. P.; Kegel, W. K.; Petukhov, A. V. Helical Colloidal Sphere Structures through Thermo-Reversible Co-Assembly with Molecular Microtubes. Angew. Chem., Int. Ed. 2013, 52, 3364-3368.

(17) de Folter, J. W. J.; Liu, P.; Jiang, L.; Kuijk, A.; Bakker, H. E.; Imhof, A.; van Blaaderen, A.; Huang, J.; Kegel, W. K.; Philipse, A. P.; Petukhov, A. V. Self-Organization of Anisotropic and Binary Colloids in Thermo-Switchable 1D Microconfinement. Part. Part. Syst. Charact. 2015, 32, 313-320.

(18) Liu, P.; de Folter, J. W. J.; Petukhov, A. V.; Philipse, A. P. Reconfigurable Assembly of Superparamagnetic Colloids Confined in Thermo-Reversible Microtubes. Soft Matter 2015, 11, 6201-6211.

(19) Jiang, L.; Peng, Y.; Yan, Y.; Deng, M.; Wang, Y.; Huang, J. Annular Ring Microtubes Formed by SDS@2 $\beta$-CD Complexes in Aqueous Solution. Soft Matter 2010, 6, 1731-1736.

(20) Ouhajji, S.; Landman, J.; Prevost, S.; Jiang, L.; Philipse, A. P.; Petukhov, A. V. In Situ Observation of Self-Assembly of Sugars and Surfactants from Nanometres to Microns. Soft Matter 2017, 13, 2421-2425.

(21) Matyjaszewski, K. Atom Transfer Radical Polymerization (ATRP): Current Status and Future Perspectives. Macromolecules 2012, 45, 4015-4039.

(22) Van Ravensteijn, B. G. P.; Kegel, W. K. Versatile Procedure for Site-Specific Grafting of Polymer Brushes on Patchy Particles via Atom Transfer Radical Polymerization (ATRP). Polym. Chem. 2016, $7,2858-2869$.

(23) Jiang, J.; Qi, B.; Lepage, M.; Zhao, Y. Polymer Micelles Stabilization on Demand through Reversible Photo-Cross-Linking. Macromolecules 2007, 40, 790-792.

(24) Trenor, S. R.; Shultz, A. R.; Love, B. J.; Long, T. E. Coumarins in Polymers: From Light Harvesting to Photo-Cross-Linkable Tissue Scaffolds. Chem. Rev. 2004, 104, 3059-3077.

(25) Cardenas-Daw, C.; Kroeger, A.; Schaertl, W.; Froimowicz, P.; Landfester, K. Reversible Photocycloadditions: A Powerful Tool for Tailoring (Nano)Materials. Macromol. Chem. Phys. 2012, 213, 144156.

(26) Mal, N. W.; Fujiwara, M.; Tanaka, Y. Photocontrolled Reversible Release of Guest Molecules from Coumarin-Modified Mesoporous Silica. Nature 2003, 421, 350-353.

(27) Kolb, H. C.; Finn, M. G.; Sharpless, K. B. Click Chemistry: Diverse Chemical Function from a Few Good Reactions. Angew. Chem., Int. Ed. 2001, 40, 2004-2021.

(28) Gübitz, G.; Schmid, M. G. Chiral Separation by Chromatographic and Electromigration Techniques. A Review. Biopharm. Drug Dispos. 2001, 22, 291-336.

(29) Aristov, M.; Eichhorn, R.; Bechinger, C. Separation of Chiral Colloidal Particles in a Helical Flow Field. Soft Matter 2013, 9, 25252530.

(30) Noguez, C.; Garzón, I. L. Optically Active Metal Nanoparticles. Chem. Soc. Rev. 2009, 38, 757-771.

(31) Matyjaszewski, K.; Gaynor, S. G.; Kulfan, A.; Podwika, M. Preparation of Hyperbranched Polyacrylates by Atom Transfer Radical Polymerization. 1 Acrylic AB* Monomers in "Living" Radical Polymerizations. Macromolecules 1997, 30, 5192-5194.

(32) Kraft, D. J.; Vlug, W. S.; van Kats, C. M.; van Blaaderen, A.; Imhof, A.; Kegel, W. K. Self-assembly of Colloids with Liquid Protrusions. J. Am. Chem. Soc. 2009, 131, 1182-1186. 
(33) van Ravensteijn, B. G. P.; Kamp, M.; van Blaaderen, A.; Kegel, W. K. General Route toward Chemically Anisotropic Colloids. Chem. Mater. 2013, 25, 4348-4353.

(34) Behl, G.; Sikka, M.; Chhikara, A.; Chopra, M. PEG-Coumarin Based Biocompatible Self-Assembled Fluorescent Nanoaggregates Synthesized via Click Reaction and Study of their Aggregation Behaviour. J. Colloid Interface Sci. 2014, 416, 151-160. 